\title{
NASA Technical Memorandum 89121
}

\author{
INVESTIGATION OF TRANSONIC REGION OF HIGH \\ DYNAMIC RESPONSE ENCOUNTERED ON AN ELASTIC \\ SUPERCRITICAL WING
}

(NASA-TM-89121) INYESTIGATICE CE TBANSCHIC

N87-20236

BEGICN OF HIGE DYHAGIC BESECASE ENCCONTERED

CH AN ELASTIC SUPERCRITICAL ING (NASA)

$13 \mathrm{p}$

CSCL 01A

G3/02 45260

DAVID A. SEIDEL

CLINTON V. ECKSTROM

MAYNARD C. SANDFORD

MARCH 1987

\section{NMSA}

National Aeronautics and

Space Administration 
INVESTIGATION OF TRANSONIC REGION OF HIGH DYNAMIC RESPONSE ENCOUNTERED ON AN ELASTIC SUPERCRITICAL WING

\author{
David A. Seidel, Clinton V. Eckstrom, and Maynard C. Sandford \\ NASA Langley Research Center
} Hampton, Virginia 23665-5225

\section{Abstract}

Unsteady aerodynamic data were measured on an aspect ratio 10.3 elastic supercritical wing while undergoing high dynamic response above Mach number of 0.90 . These tests were conducted in the NASA Langley Transonic Dynamics Tunnel. A previous test of this wing predicted an unusual instability boundary based upon subcritical response data. During the present test no instability was found, but an angle of attack dependent narrow Mach number region of high dynamic wing response was observed over a wide range of dynamic pressures. The effect on dynamic wing response of wing angle of attack, static outboard control surface deflection and lower surface spanwise fence located near the 60 percent local chordline was investigated. The driving mechanism of the dynamic wing response appears to be related to chordwise shock movement in conjunction with flow separation and reattachment on both the upper and lower surfaces.

\section{Nomenclature}

$C_{L} \quad$ lift coefficient

$C_{p} \quad$ pressure coefficient

h altitude, feet

$\mathrm{Hz}$ Hertz, cycles/second

M free-stream Mach number

q free-stream dynamic pressure, psf

$R \quad$ Reynolds number, per foot

$x / c$ fraction of local chord

a wing root angle of attack, degrees (positive leading-edge up)

om mean control surface deflection angle, degrees (positive trailing edge down)

$\Delta C_{p} \quad$ lifting pressure coefficient (positive

up)

$n$ fraction of semispan

\section{Introduction}

Single degree-of-freedom bending mode oscillations have unexpectedly been encountered during experiments with several aircraft configurations. These oscillations are characteristically large amplitude oscillations in a low frequency vibration mode and tend to be angle of attack dependent. These oscillations have been observed on a low aspect ratio wing with subsonic airfoil shape, on the B-1A during a wind-up turn, ${ }^{2}$ on the canard of the HIMAT aeroelastic model at negative angle of attack ${ }^{2}$ and on a forward swept wing force model wing panel at a negative angle of attack. ${ }^{2}$ Linear theory flutter analysis was unable to predict any of these oscillations. The $B-1$ instability has been attributed to a dynamic leading edge vortex flow mechanism. The other instabilities are attributed to transonic shock wave motion coupling with the first wing bending mode.

An unusual transonic instability also was encountered near $M=0.9$ during an unsteady pressure test of the second Aeroelastic Research Wing $(A R W-2)^{3}$ of the NASA Drones for Aerodynamic and Structural Testing (DAST) program. ${ }^{3}, 4$ This aeroelastic supercritical wing was initially tested in October 1983 in the Langley Transonic Dynamics Tunnel (TDT). The wing had an aspect ratio of 10.3 and a leading edge sweepback angle of $28.8^{\circ}$. The wing also had a hydraulically driven outboard trailing-edge control surface and was instrumented with dynamic pressure transducers. This unusual transonic instability boundary was predicted using a subcritical response technique. This instability was predicted to occur at an almost constant Mach number of 0.9 for all dynamic pressures tested. The wing motion was primarily first wing bending mode response and was angle of attack dependent. Identifying such an unusual transonic instability boundary appears to be beyond the scope of linear theory analysis. ${ }^{4}$

Thus a second wind tunnel test was performed on the DAST ARW-2 wing to further investigate this unusual instability. The primary purpose of the test was to establish firmly the existence of the instability boundary and to gather wing response data and dynamic pressure measurements to help understand the mechanism forcing the wing oscillations.

This paper presents the results from this second wind tunnel test of the DAST ARW-2 wing. Dynamic wing motion and pressures were measured for wind tunnel Mach numbers from 0.50 to 0.96 at dynamic pressures from 100 to over 340 pounds per square foot (psf). Wool tufts were used to visualize the flow patterns on the wing in the instability region. The effect on dynamic wing response of wing angle of attack, static outboard control surface deflection and a spanwise fence on the lower surface were investigated. 
Model

Figure 1 shows the wing and fuselage configuration mounted in the wind tunnel. The elastic semispan wing used in the present study is the DAST ARW-2 right wing panel. A half-body fuselage was used to simulate the drone fuselage. This fuselage had shorter nose and tafl sections than does the drone fuselage since no supersonic tests were to be made. The center section of the fuselage was similar to the actual drone fuselage in both diameter and wing location to generate the proper airflow over the inboard section of the wing. Both the fuselage and the wing were mounted on a remotely controlled turntable mechanism located on the tunnel sidewall.

The wing planform is shown in figure 2. The wing had an aspect ratio of 10.3 with a leading edge sweep angle of $28.8^{\circ}$. The wing was equipped with three hydraulically driven control surfaces, two inboard and one outboard. For this test, the inboard surfaces were held fixed at $0^{\circ}$ deflection and only the outboard surface was deflected statically. The outboard surface hinge line was located at 77 percent of local chord.

The wing contour was formed from three different supercritical airfoils as described in ref. 5. These three airfoils were located at the following spanwise wing stations: the wing-fuselage junction $(n=0.071)$, the wing planform break ( $n=0.426$ ) and the wing tip ( $n=$ $1.000)$ and had thickness-to-chord ratios of $0.146,0.120$ and 0.106 , respectively. The three supercritical airfoll shapes and wing twist were defined for the design cruise condition and are described in ref. 6. The wing design shape was defined by using straight line interpolation along constant percent chords between these three airfoil sections for the design cruise conditions of $M=0.80, C_{L}=0.53\left(\alpha=1.3^{\circ}\right)$ and $h=46,800$ feet $(q=127 \mathrm{psf})$. The jig shape for fabrication of the flexible wing was determined by first calculating the wing load distribution at the design cruise conditions. The second step was to calculate the wing deflection and twist for the wing load distribution using a finite element structural model of the wing. The final step was to subtract the calculated wing deflection and twist from the wing design shape to define the wing jig shape.

The wing primary structure consisted of a front spar at 25 percent of local chord and a rear spar at 62 percent of local chord. ${ }^{6}$ Ribs were placed perpendicular to the rear spar every 13.2 inches except for the outboard wing tip rib which also served as a spar end fitting. The spars and ribs were machined from 7075-173 aluminum alloy. The wing skin was made of fiberglass material with honeycomb panels sandwiched between the middle two layers of fiberglass for areas of skin not located over the spars or ribs. The number of layers of fiberglass used to make the skin varied from 36 at the inboard end to 27 at the outboard end with approximately 25 percent of the layers at \pm 45 degrees orientation.
Also shown in Figure 2 are the locations of the wing instrumentation. The instrumentation consisted of 191 dynamic pressure transducers and 10 accelerometers. In addition, strain gauge bridges were located near the wing root to measure bending moments. Differential pressure gauges were mounted in each supply line to the hydraulic actuators of each control surface to measure hinge moments. Small potentiometers were used to measure the control surface angular displacement. The model angle of attack was measured by a servo accelerometer that was mounted near the wing root. Both steady and unsteady pressures were obtained using differential pressure transducers referenced to the tunnel's static pressure. Streamwise rows of upper and lower surface pressure orifices were located at six span stations: $n=0.274$, $0.476,0.599,0.707,0.871$ and 0.972 . The fifth row at $n=0.871$ lies along the mid-span of the outboard control surface. All of these surface orifices were connected to pressure transducers by matched tubes having an inner diameter of 0.040 inch and a length of 18 inches. In order to determine the tube transfer functions needed to correct the unsteady pressure data from these matched-tube transducers, simultaneous measurements were also obtained from a row of in situ transducers mounted on the wing upper surface at $n=0.875$, parallel to the fifth row of surface orifices. Dynamic wing deflections were determined using the 10 accelerometers.

\section{Wind Tunnel}

The Langley Transonic Dynamics Tunnel (TDT) is a closed-circuit continuous-flow tunnel which has a 16-foot square test section with slots in all four walls. Mach number and dynamic pressure can be varied simultaneously, or independently, with either atr or Freon as a test medium. Freon was used for the tests in this investigation.

\section{Data Acquisition and Analysis}

Data from the model instrumentation were acquired using the TDT real-time data acquisition system. ${ }^{7}$ The pressure data were acquired using the electronically scanned pressure (ESP) system. ${ }^{8}$ The ESP system is a sequential, digital pressure sampling system equivalent to a mechanical scani-valve. A11 data were digitized in real-time and written on magnetic tape for later analysis. The three inboard rows of surface orifices were digitized at 31.25 samples per second while the three outboard rows were digitized at 250 samples per second. All other data, including model and tunnel conditions and wing accelerometers, were digitized at 1000 samples per second.

Static pressures were recorded for all six rows of surface orifices. Each pressure signal was averaged for 300 samples to acquire its mean value. Wing bending moments were measured for all cases where static pressures were recorded. The bending moment measurements were averaged for 0.3 seconds to obtain a mean value. Dynamic time histories were recorded for the three outboard rows of surface orifices and all in wing accelerometers. The dynamic time histories 
were recorded for a minimum of 15 seconds at each flow condition.

\section{Previous Test Results}

During the first test of the ARW-2 wing in the TDT an unusual wing instability, with motion similar to the wing first bending mode, was encountered. ${ }^{4}$ The boundary was determined for a wing angle of attack and control surface deflection of $0^{\circ}$ within the wind tunnel limits as shown in Figure 3. Also shown in Figure 3 as a solid line is the predicted linear theory (doublet lattice theory) flutter boundary, which is of a conventional nature, exhibiting a drop in the dynamic pressure at flutter as Mach number increases. Typicaliy, experimental flutter boundaries attain a minimum value of dynamic pressure near $M-1.0$ followed by rapid rise in the flutter dynamic pressures as speed increases further. This is in contrast with the experimental instability boundary shown in Figure 3, which is nearly vertical at a Mach number of 0.90 . The measured boundary was determined using a familiar subcritical response technique known as peak-hold. ${ }^{9}$ The peak-hold results definitely showed indication of instability onset. However, the normal verification procedure of obtaining hard instability (zero damping) points was avoided for fear of damaging the model which was to be used in future flight programs. The boundary was predicted to occur at a nearly constant Mach number of 0.90 beginning at a low dynamic pressure of about 50 pounds per square foot (psf) $(R=874,000)$ and rising nearly vertical to over 300 psf $(R=5,300,000)$. The observed wing motion during the instability was similar to the wing first bending mode, the frequency of which was measured to be $8.3 \mathrm{~Hz}$ in the wind-off model vibration tests. The instability frequency was $8.6 \mathrm{~Hz}$ at the lowest dynamic pressure point and increased with dynamic pressure to about $13 \mathrm{~Hz}$ at the highest dynamic pressure point. It is interesting to note that the predicted flutter frequency (using doublet lattice linear theory aerodynamics) was $24.3 \mathrm{~Hz}$ at a Mach number of 0.80 . An attempt to predict the instability using a three-dimensional transonic small disturbance code was unsuccessful as described in ref. 10.

Because of recent interest in angle of attack effects and shock induced effects on wing instabilities, ${ }^{11}$ several additional test runs were made. These runs included variation of the wing angle of attack as the predicted instability boundary was approached, comparison using air or Freon as the test medium and comparison with and without a transition strip near the wing leading edge. The instability was found to be sensitive to variation in angle of attack and, generally, the minimum damping occurred at or near zero wing root angle of attack. In Figure 3 the solid symbol indicates the Mach number and dynamic pressure where the comparison tests were made. The results showed no significant difference in the instability boundary for tests in air or Freon. Reynolds number values in Freon are approximately 3.1 times greater than those obtained in air. There were also no significant differences for tests in Freon with or without a transition strip.

\section{Present Test Results and Discussion}

Dynamic pressures and wing deflections were measured for a large number of test conditions in the TDT using Freon as a test medium. Data were taken at Mach numbers from 0.5 to 0.96 for two stagnation pressures. For a Mach number range of $0.8-0.96$ the two stagnation pressures gave a dynamic pressure variation of $125-166$ psf and 260 - 340 psf. These two stagnation pressures will be referred to as the low and high density conditions.

\section{Dynamic Wing Response}

Dynamic wing response variations as a function of Mach number are presented to examine the effects of changing density conditions and model angle of attack. Also, the effect of static outboard control surface deflection and the addition of a spanwise fence on the lower surface are examined. All dynamic wing response data presented is obtained from the rear wing tip accelerometer.

Mach Number and Dynamic Pressure Effects: Figure 4 shows the peak-hold results from the wing tip accelerometer for both the low and high density conditions. The wing angle of attack and control surface deflection were held at $0^{\circ}$. The data show that no instability was found but instead a region of high dynamic wing response was observed. For the lower density condition $(q=125-166$ psf) the wing motion reaches a maximum at $M=0.93$ and then rapidly decreases with increasing Mach number. The same trend occurs for the higher density condition $(q=260$ - 340 psf) with maximum wing motion occurring near $M=0.92$. The observed wing tip maximum dynamic amplitudes are noted in Figure 4. At the lower density condition, the amplitude of the wing tip motion was approximately 2 inches peak-to-peak. At the higher density condition, which has double the dynamic pressure, the amplitude of the wing tip motion doubled to approximately 4 inches peak-to-peak.

Aiso shown in Figure $4(b)$ at $M=0.92$ is a single point for $\alpha=-1^{\circ}$ where 6 inches peak-to-peak amplitude of wing tip motion was observed. At this condition the wing motion was so severe that the tunnel bypass valves were opened to rapidly reduce the dynamic pressure and associated wing motion.

Angle of Attack Effects: Figure 5 shows the peak-hold results from the wing tip accelerometer for three wing angles of attack at the lower density condition. The mean control surface deflection was held at $0^{\circ}$. As shown in the figure, the maximum dynamic wing response occurred for a wing angle of attack of $0^{\circ}$ at $M=$ 0.93. Changing the wing angle of attack to 2 and -2 degrees decreased the maximum wing response and shifted the corresponding Mach number to 0.94. Similar angle of attack trend results were seen at the higher density condition. Data were taken for wing angles of attack of 1,0 and -1 degrees up to $M=0.9$ and fell within the scatter of the experimental data observed at $0^{\circ}$ as shown in Figure 4. Therefore it is believed that for this configuration the 
maximum wing response occurs when the wing angle of attack is nominally at $0^{\circ}$.

Control Surface Effects: Figure 6 shows the peakthold results from the wing tip accelerometer for three outboard control surface mean deflection angles at the lower density condition. The wing angle of attack was set at $0^{\circ}$. The figure shows a small increase in wing response for the control surface deflection of $6^{\circ}$ (trailing edge down). However, a significant reduction in wing response is shown for a deflection of $-6^{\circ}$. The wing tip maximum response peak is reduced by half and shifted to a lower Mach number of 0.91 .

Lower Surface Spanwise Fence Effects: In an at tempt to disturb the flow and change the dynamic wing response, a $1 / 2$-inch high spanwise fence was attached to the lower surface at approximately the $60 \%$ local chordline as shown in figure 7. The fence ran from the wing planform break $(n=0.426)$ to within 5 inches of the wing tip $(n=0.956)$. The fence was made up of 5 separate one foot-long pieces of aluminum placed end to end to minimize increasing the wing stiffness. The effect of the fence on the wing tip accelerometer peak hold response at the lower density condition is shown in Figure 8 . The fence has a significant effect upon the wing response, lowering the amplitude of maximum wing motion and shifting the peak value to a lower Mach number of 0.90 .

\section{Mean Pressures}

Figure 9 shows the mean chordwise pressure distribution at the $87.1 \%$ span station for nine Mach numbers at the lower density condition. The wing angle of attack and outboard mean control surface deflection were $0^{\circ}$. As Mach number increases, a shock develops on the upper surface at $M=0.85$ and becomes quite strong at $M=0.89$. The criteria used to determine trailing edge flow separation from mean pressure measurements is the attainment of negative pressure coefficients at the $95 \%$ chord location. When negative pressures are sustained aft of this location, the flow is considered to be separated. Based upon the mean pressure distributions shown in Figure 9 , it appears that flow separation on the upper surface is evident at $M=0.92$ and is established strongly at $M=$ 0.94 . The lower surface develops a strong shock at $M=0.92$ and the pressure distributions indicate flow separation at $M=0.96$.

\section{Flow Visualization}

Wool tufts were placed on the upper and lower wing surfaces for several test runs to visualize the flow patterns on the wing. The tufts were placed on eight span stations located at $\eta=.517, .558, .635, .671, .761, .816, .905$ and .938 , as shown in Figure 10. The tufts were one inch long and on the six inboard span stations were located at every $10 \%$ of local chord. On the two outboard span stations the tufts were located between 10 and $90 \%$ chord at every $20 \%$ of local chord.

Table 1 lists the regions of separated flow on the wing as indicated by the tuft data for Mach numbers from 0.85 to 0.96 at the lower density condition. Upper surface flow separation is first indicated at $M=0.88$. The region of separated flow expands upstream and outboard as Mach number increases to 0.94 and then remains constant to $M=0.96$. Flow separation on the lower surface is initially indicated at $M=0.90$. The region of separated flow expands upstream and outboard as Mach number increases to 0.94 . At $M=0.96$ the region of separated flow on the lower surface decreases, moving downstream and inboard.

Comparing this data to the mean pressure distributions shown in figure 9 leads to two conclusions. The first is that the mean pressure data gives an incomplete picture of the flow separation. The mean pressure data, taken at $n=0.871$, does not indicate flow separation on the upper surface until $M=0.92$ while the wool tufts indicate separation in the region of

Table 1. Separated flow regions as shown by wool tufts

\begin{tabular}{|c|c|c|c|c|}
\hline \multirow[b]{3}{*}{ M } & \multicolumn{4}{|c|}{ Region of separated flow } \\
\hline & \multicolumn{2}{|c|}{ Upper surface } & \multicolumn{2}{|c|}{ Lower surface } \\
\hline & $x / c$ & $\eta$ & $x / c$ & $n$ \\
\hline .85 & $-\cdots-$ & $\cdots-$ & $-\cdots$ & - \\
\hline .88 & $.8-1 . n$ & $.517-.816$ & $\cdots$ & $-\ldots$ \\
\hline .90 & $.7-1.0$ & $.517-.905$ & $.6-1.0$ & $.635-.761$ \\
\hline .92 & $.7-1.0$ & $.517-.938$ & $.6-1.0$ & $.635-.938$ \\
\hline .94 & $.6-1.0$ & $.517-.938$ & $.5-1.0$ & $.635-.938$ \\
\hline .96 & $.6-1.0$ & $.517-.938$ & $.6-1.0$ & $.635-.905$ \\
\hline
\end{tabular}


the pressure transducers near $M=0.89$. Flow separation on the lower surface is not indicated by the mean pressures until $M=0.96$ while the tufts indicate separation in the region at $M=$ 0.92. The second conclusion is that flow separation, as shown by the tuft data, coincides with the occurrence of strong shocks on a surface, as shown by the mean pressure data in Figure 9. This flow separation occurs near $M=$ 0.89 on the upper surface and $M=0.92$ on the lower surface at the $87.1 \%$ span station.

\section{Wing Deflection and Twist}

During the test, measurements of the mean wing tip deflection and twist were made using an optical cathetometer instrument focused on a straight line drawn on the outboard tip of the wing. The results of the wing tip measurements for the lower density condition at a wing angle of attack and mean control surface deflection of $0^{\circ}$ are shown in Figure 11. The wing tip deflection and twist increase as Mach number increases up to a maximum near $M=0.85$. At higher Mach numbers the wing tip deflection and twist values decrease rapidly as the Mach number increases. This agrees with the tuft data which shows flow separation beginning on the upper surface at $M=0.88$, causing loss of lift (see Figure 9) and the resulting decrease in wing deflection and twist.

\section{Instantaneous Pressures}

Figure 12 shows the instantaneous chordwise pressure distribution at the $87.1 \%$ span station for $M=0.92, a=-1^{\circ}$ and $\delta_{m}=0^{\circ}$. This is the condition at which 6 inch peak-to-peak wing tip motion occurred (Figure 4(b)). The instantaneous pressure distributions are shown for the maximum and minimum vertical wing tip deflection. Based upon the pressure at $95 \%$ chord, at the maximum wing tip deflection the flow aft of the shock is separated on the upper and lower surfaces. The flow is attached on both surfaces when the vertical tip deflection is a minimum.

This figure points out an important feature of this dynamic motion. At conditions where large amplitude dynamic motion is encountered the trailing-edge flow begins a pattern of separating and reattaching on the wing, which coincides with the shock wave motion. As the Mach number is increased above 0.92 the flow behind the shock remains separated (see Figure 9) and the amplitude of the motion rapidly decreases (see Figure 4). Thus it appears that the dynamic wing response is related to chordwise shock motion in conjunction with shock induced flow separation and reattachment on both the upper and lower surfaces. This conclusion is supported further by the results obtained when the spanwise fence was attached to the wing lower surface. The fence prevented reattachment of the flow on that surface and the maximum wing motion was found to be dramatically reduced as shown in Figure 8 .

The alternating separation and reattachment of the flow on the upper and lower surfaces also explains the discrepancy between the mean pressure and wool tuft data. The mean pressure data gives an average of the pressure values in the trailing edge region. If, on the average, the flow is attached most of the time, the mean pressure distributions will indicate that the flow is attached. The mean data gives an accurate indication of separation only when the flow remains separated most of the time. Another point to note is that while the wool tufts indicate flow separation, they are inadequate for indicating the subsequent flow reattachment.

Figure 13 shows a time history of upper and lower surface pressures at the same span station and flow conditions as given in Figure 12. All pressures are arbitrarily plotted so that they fit near each other. However, the last chordwise pressures on both surfaces are plotted with a zero reference line. For the last chordwise pressures, the figure clearly shows the separation and reattachment of flow in the trailingedge region of both surfaces as the pressure values fluctuate above and below zero. The shock motion, as indicated by large pressure variations, is also shown in the figure. For example, the upper surface shock can be seen to move from in front of $68.0 \%$ of local chord to behind $74.2 \%$. The lower surface shock moves from in front of $46.0 \%$ to behind $51.3 \%$. At the bottom of the figure the measured wing root bending moment time history is plotted for reference. For the observed motion, the wing root bending moment is proportional to wing tip displacement, being maximum for maximum positive (up) wing tip position.

\section{Concluding Remarks}

Unsteady aerodynamic and response data were measured on an aspect ratio 10.3 elastic supercritical wing undergoing high dynamic response above Mach number of 0.90 . The wing had been tested previously in the NASA Langley TDT and an unusual instability boundary was predicted based upon subcritical response data. Contrary to the predictions, no instability was found during the present test. Instead a region of high dynamic wing response was observed which reached a maximum value between Mach numbers 0.92 and 0.93 . The amplitude of the dynamic response increased directly with dynamic pressure. Maximum wing tip motion observed was 6 inches peak-to-peak. The dynamic wing response was sensitive to angle of attack, with maximum motion occurring near $\alpha=0^{\circ}$. Static deflection of the outboard control surface was found to decrease significantly the dynamic response for $\delta_{m}=-6^{\circ}$. A spanwise fence installed on the lower surface at approximately the $60 \%$ local chordine to disturb the flow pattern resulted in a significant decrease in dynamic wing response.

The response appears to be related to chordwise shock movement in conjunction with flow separation and reattachment on the upper and lower wing surfaces. At Mach numbers above 0.93 the dynamic response rapidly decreases with increasing Mach number. This is likely due to the flow remaining completely separated aft of the shock. Mean pressure data indicates separation only after the flow has become fully 
separated. Tufts on the wing indicated the onset of flow separation at slightly lower Mach numbers than indicated by the mean pressure data. The onset of flow separation coincided with the occurrence of strong shocks on a surface. Instantaneous pressure distributions indicated that the flow was intermittently separating and reattaching near the trailing edge under conditions of maximum wing motion.

\section{References}

1Erickson, L. L.: Transonic Single-Mode Flutter and Buffet of a Low Aspect Ratio Wing Having a Subsonic Airfoil Shape. NASA TN D-7346, January 1974.

Dobbs, S. K.; Miller, G. D.; and Stevenson, J. R.: Self-Induced Oscillation Wind Tunnel Test of a Variable Sweep Wing. AIAA Paper No. 85-0739-CP, April 1985.

Murrow, H. N.; and Eckstrom, C. V.: Drones for Aerodynamic and Structural Testing (DAST) - A Status Report. Journal of Aircraft, Vol. 16, No. 8, August 1979, pp. 521-526.

"Seidel, D. A.; Sandford, $M_{\text {. }} C_{\text {. }}$; and Eckstrom, C. V.: Measured Unsteady Transonic Aerodynamic Characteristics of an Elastic Supercritical Wing with an Oscillating Control Surfaçe. AIAA Paper No. 85-0598-CP, April 1985. Byrdsong, T. A.; and Cuyler, W. B., Jr. Wind-Tunnel Investigation of Longitudinal and Lateral-Directional Stability and Control Characteristics of a 0.237-Scale Model of a Remotely Piloted Research Vehicle With a Thick, High-Aspect-Ratio Supercritical Wing. NASA TM 81790 , July 1980.

Eckstrom, C. V.: Loads Calibration of Strain Gage Bridges on the MAST Project Aeroelastic Research Wing (ARW-2). NASA TM 87677 March 1986.

Cole, P. H.: Wind Tunnel Real-Time Data Acquisition System. NASA TM 80081, April 1979.

Chapin, W. G.: Dynamic-Pressure Measurements Using an Electronically Scanned Pressure Module. NASA TM 84650, July 1983.

${ }^{9}$ Sandford, M. C.; Abel, I.; and Gray, D. L.: Development and Demonstration of a Flutter-Suppression System Using Active Contrgls. NASA TR R-450, December 1975.

Bennett, R. M.; Seidel, D. A.; and Sandford, M. C.: Transonic Calculations for a Flexible Supercritical Wing and Comparison with Experiment. AIAA Paper No. 85-0665-CP, April 1985

'Yates, E. C., Jr.; Wynne, E. C.; and Farmer, M. G.: Effects of Angle of Attack on Transonic Flutter of a Supercritical Wing. Journal of Aircraft, Vol. 20, No. 10, October 1983, pp. 841-847.

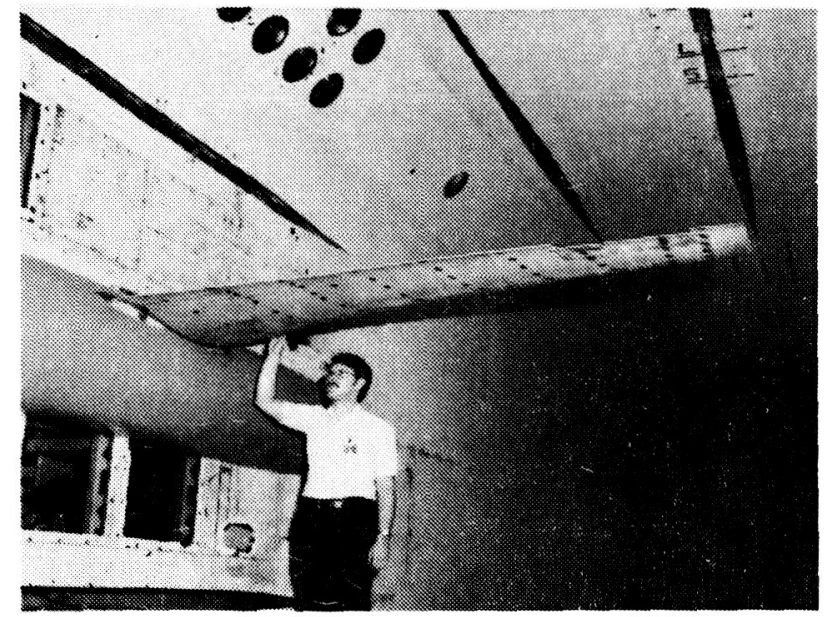

Fig. 1 Wing mounted in TDT test section.

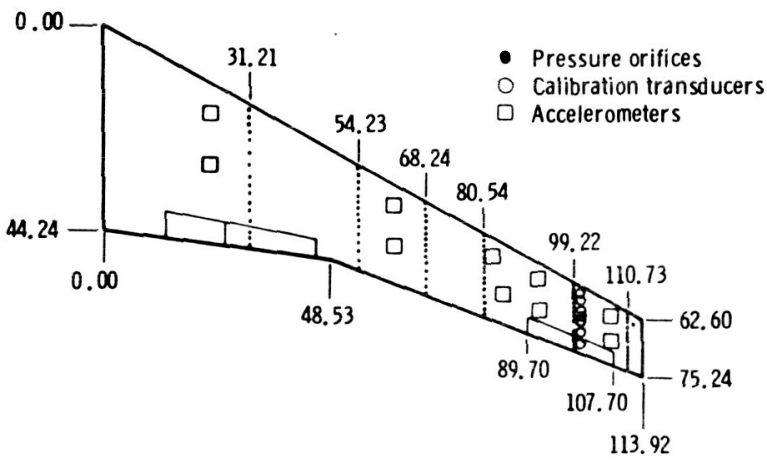

Fig. 2 Wing planform and instrumentation locations (in inches).

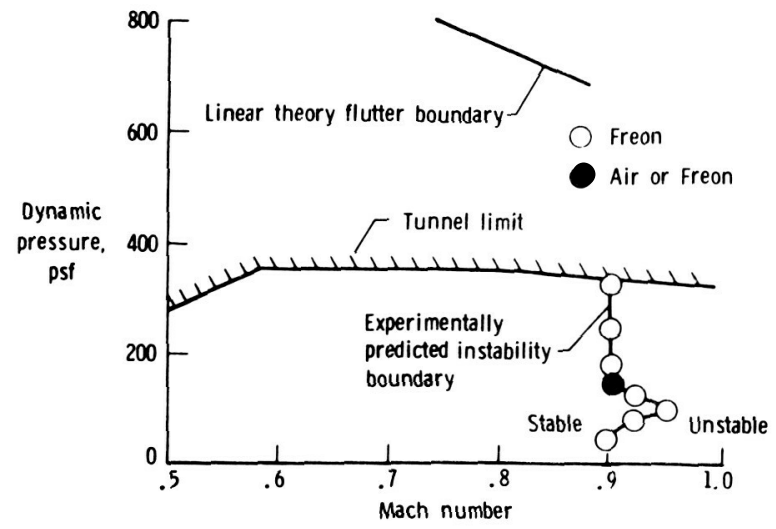

Fig. 3 Experimental and linear theory predictions of wing instability boundary. 


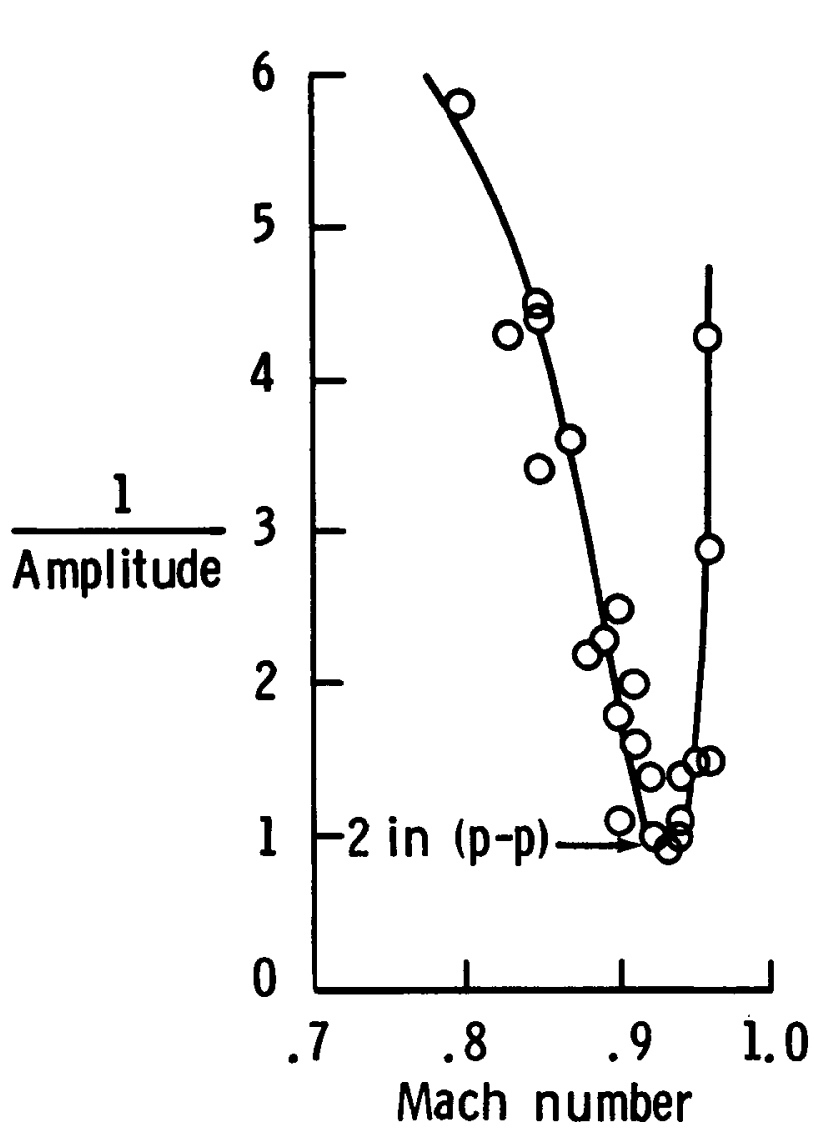

(a) $q=125-166$ psf.

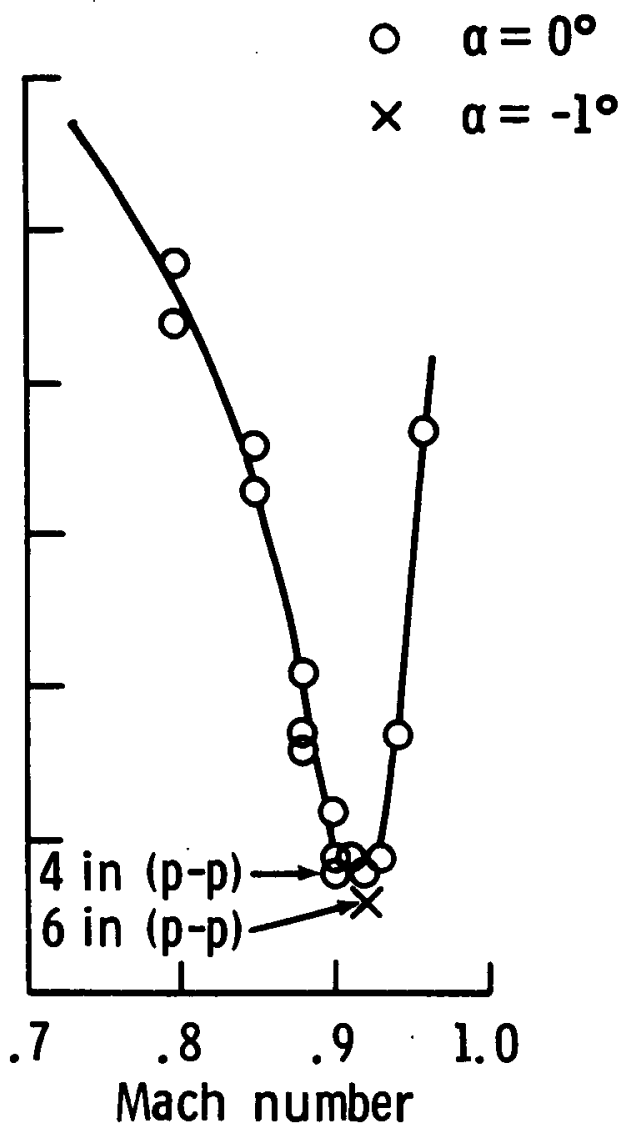

(b) $q=260-340$ psf.

Fig. 4 Peak-hold response data from wing tip accelerometer at $\delta_{m}=0^{\circ}$.

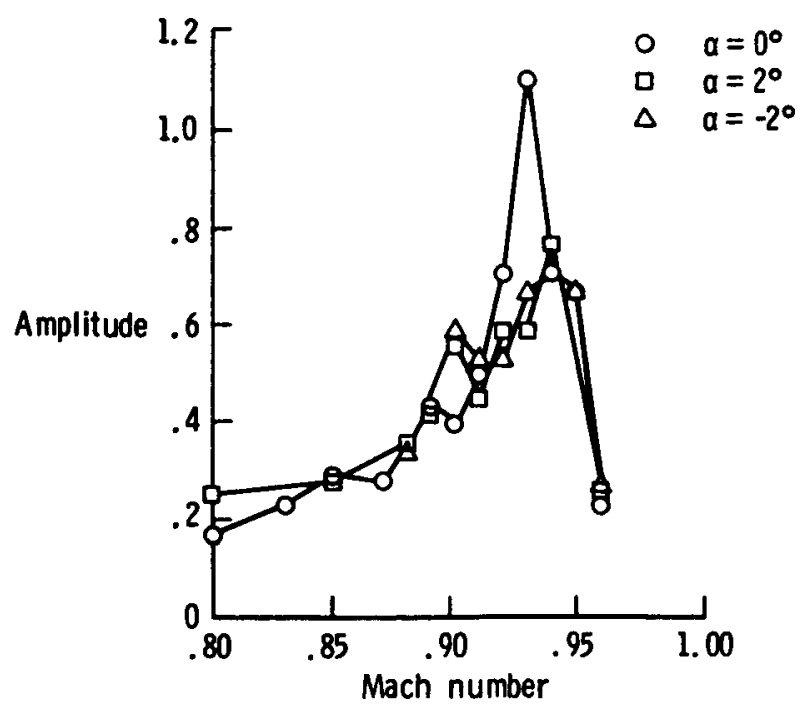

Fig. 5 Peak hold response data from wing tip accelerometer for three angles of attack at $\delta_{\mathrm{m}}=0^{\circ}$ and $q=125$ 166 psf.

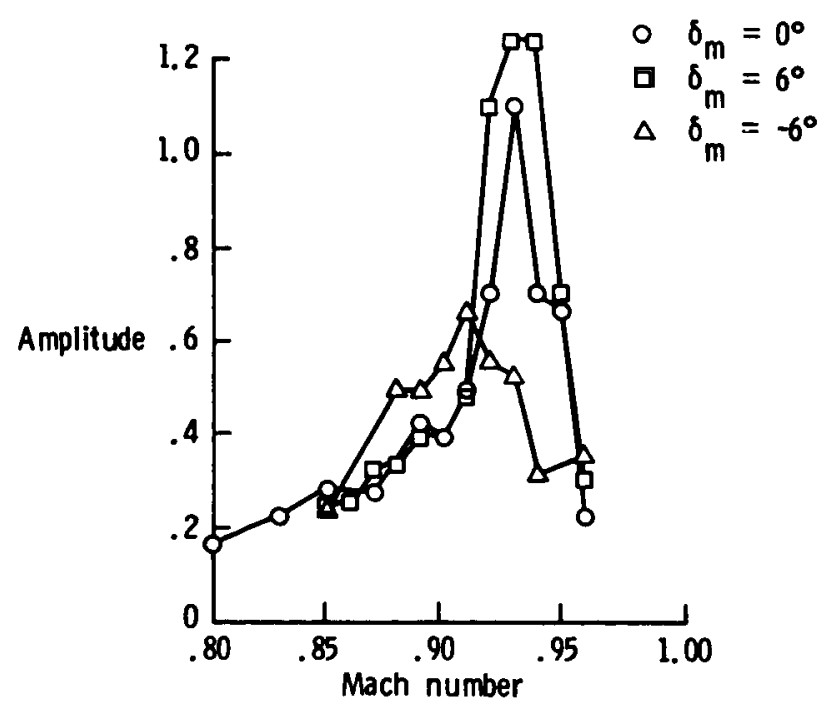

Fig. 6 Peak-hold response data from wing tip accelerometer for three mean control surface deflection angles at $\alpha=0^{\circ}$ and $q=125-166$ psf. 


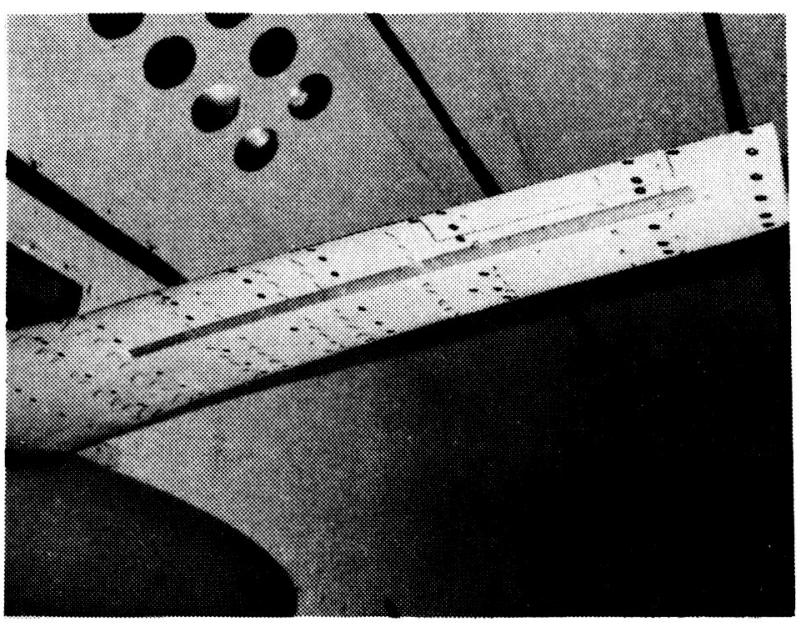

Fig. 7 Wing with spanwise fence attached to lower surface.

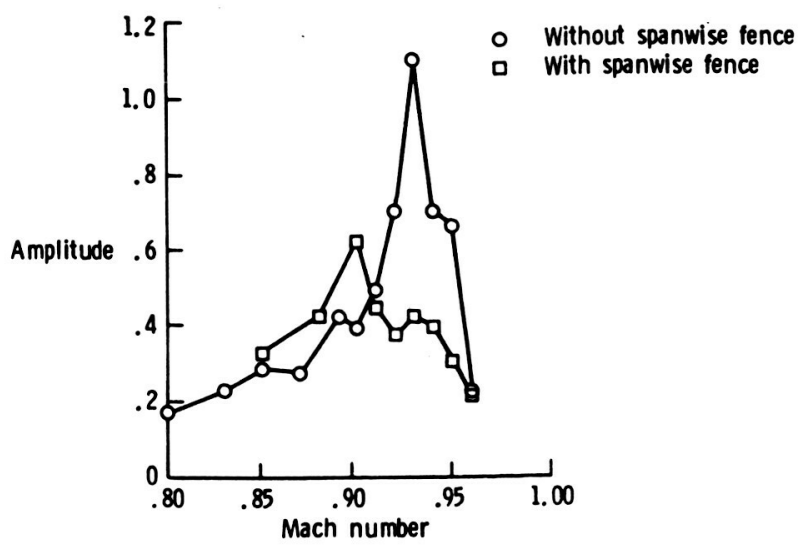

Fig. 8 Peak-hold response data from wing tip accelerometer for wing with and without lower surface spanwise fence at $\alpha=0^{\circ}, \delta_{m}=0^{\circ}$ and $q=125$. 166 psf.

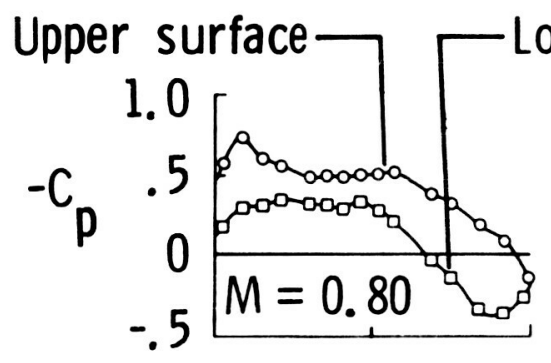

Lower surface
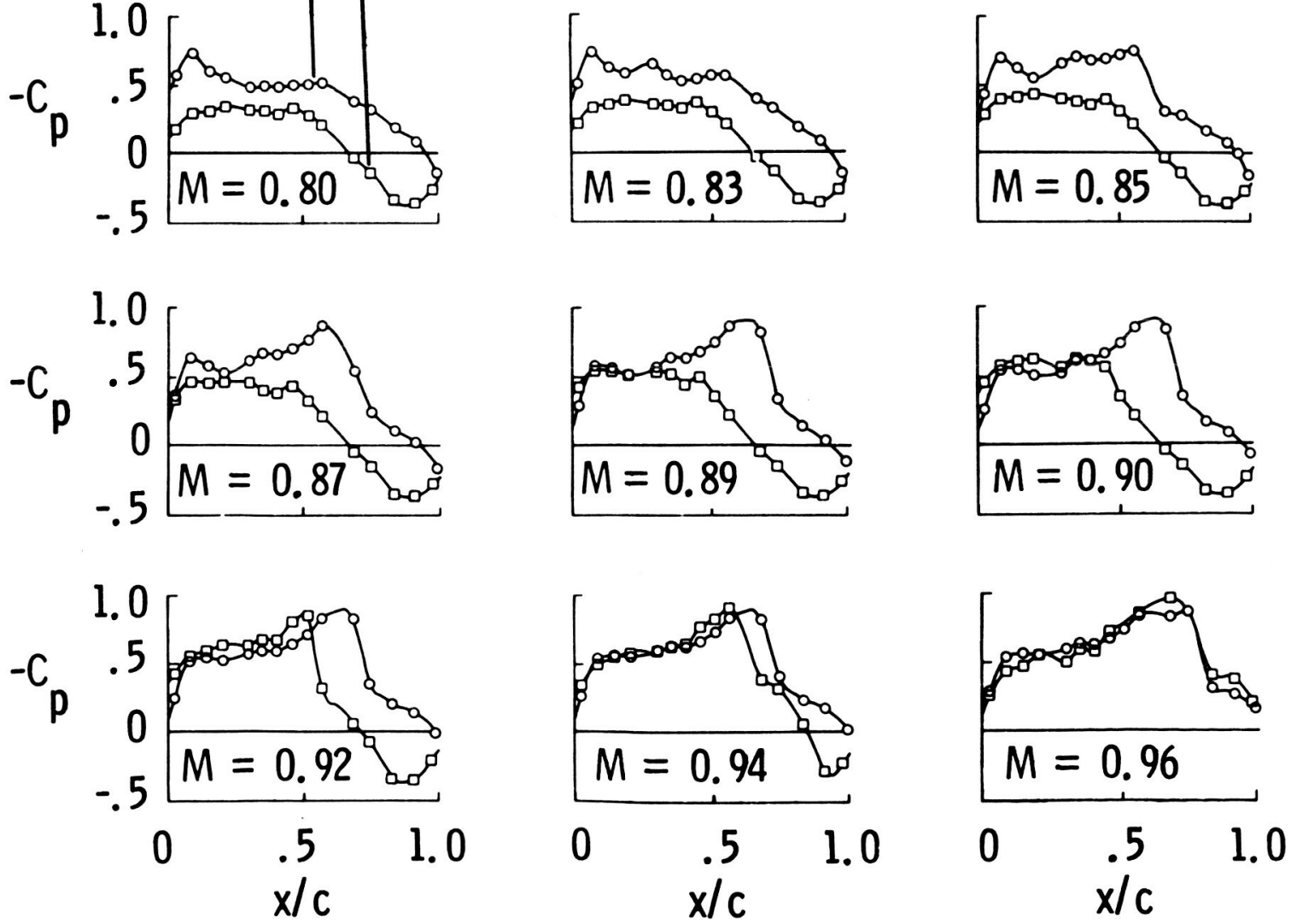

Fig. 9 Mean chordwise pressure distributions for nine Mach numbers at $\eta=0.871, \alpha=0^{\circ}, \delta_{m}=0^{\circ}$ and $q=125-166$ psf. 


\section{OF POOR QUALTY}

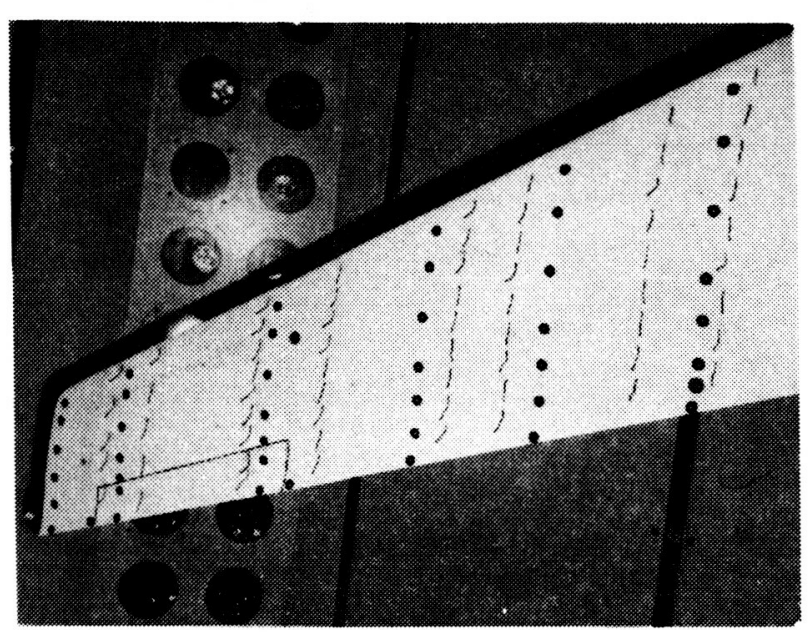

Fig. 10 Wool tufts installed on wing lower surface.
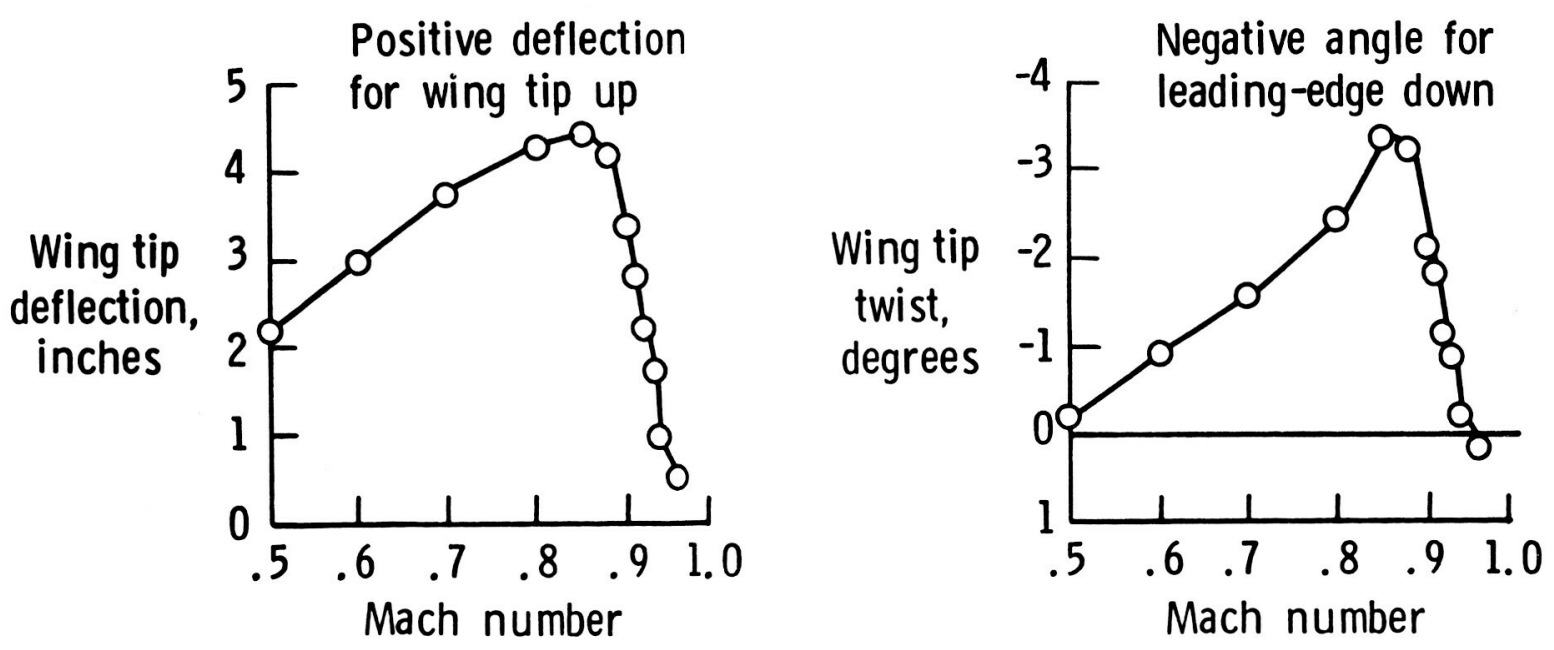

Fig. 11 Measured mean wing tip deflection and twist versus Mach number at $\alpha=0^{\circ}, \delta_{m}=0^{\circ}$ and $q=100-166$ psf. 
O Maximum deflection $\mapsto$

- Minimum deflection $\mapsto$
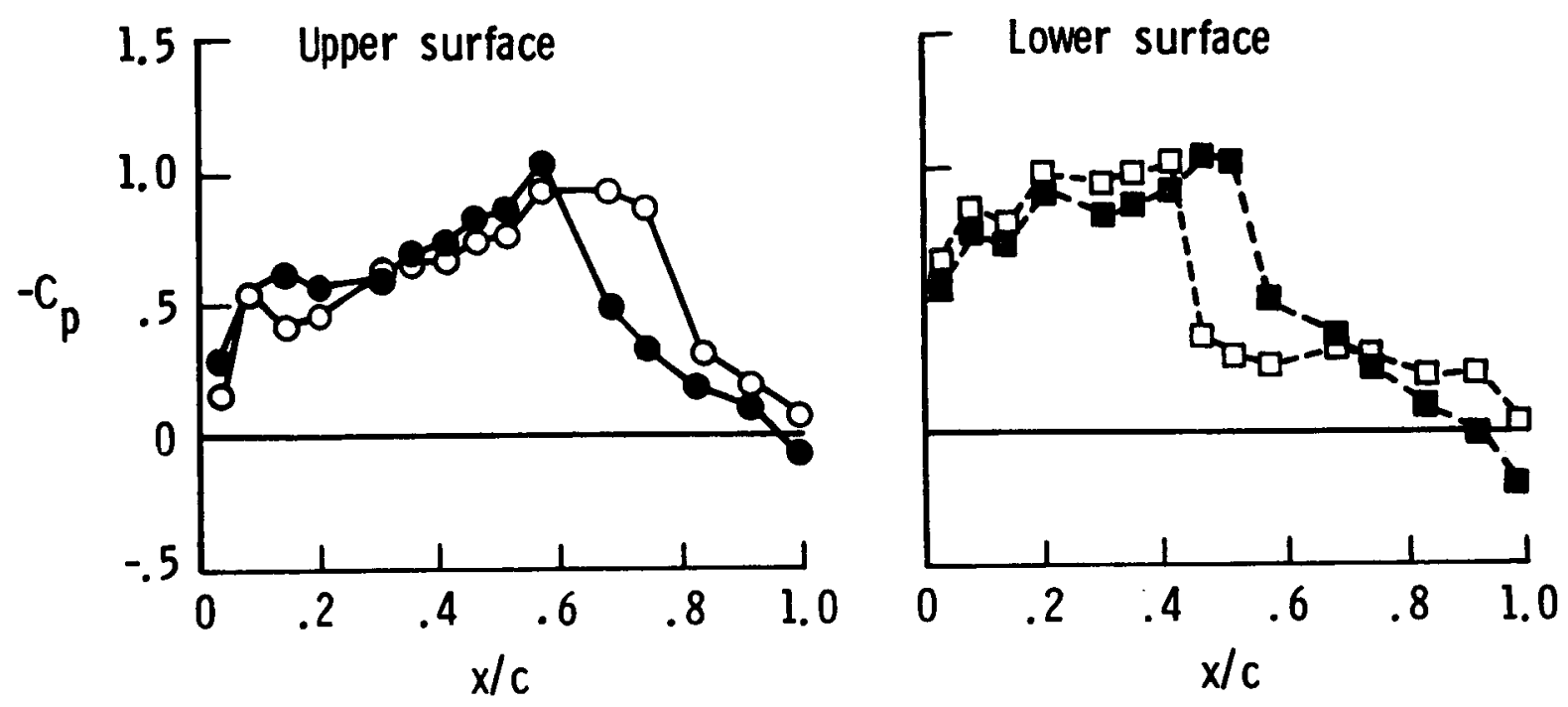

Fig. 12 Instantaneous chordwise pressure distributions at maximum and minimum wing tip deflection at $\eta=0.871, M=0.92, \alpha=-1^{\circ}$ and $\delta_{m}=0^{\circ}$.

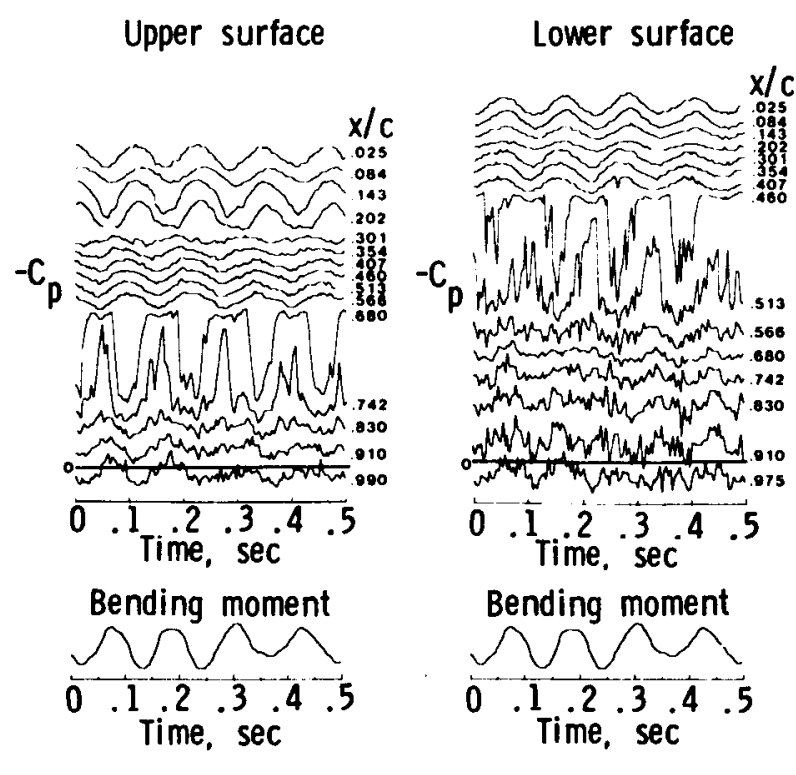

Fig. 13 Pressure variation with time at

$n=0.871, M=0.92, \alpha=-1^{\circ}$ and

$\delta_{m}=0^{\circ}$. 


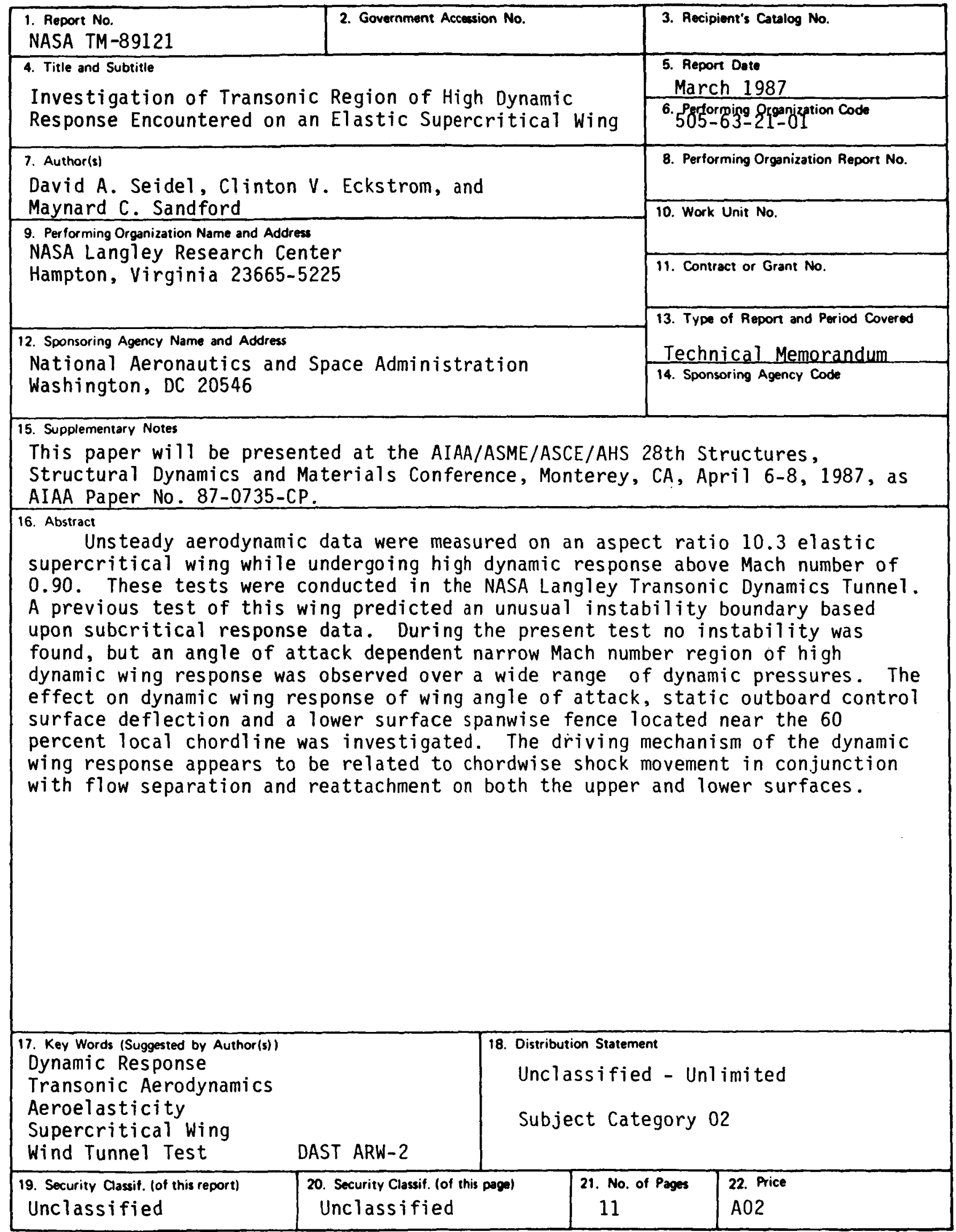

